\title{
Carleson measures and uniformly discrete sequences in strongly pseudoconvex domains
}

\author{
Marco Abate ${ }^{1}$, Alberto Saracco ${ }^{2}$
}

November 2009

\begin{abstract}
We characterize using the Bergman kernel Carleson measures of Bergman spaces in strongly pseudoconvex bounded domains in $\mathbb{C}^{n}$, generalizing to this setting theorems proved by Duren and Weir for the unit ball. We also show that uniformly discrete (with respect to the Kobayashi distance) sequences give examples of Carleson measures, and we compute the speed of escape to the boundary of uniformly discrete sequences in strongly pseudoconvex domains, generalizing results obtained in the unit ball by Jevtić, Massaneda and Thomas, by Duren and Weir, and by MacCluer.
\end{abstract}

\section{Introduction}

In his celebrated solution of the corona problem in the disk, Carleson $[\mathrm{C}]$ introduced an important class of measures to study the structure of the Hardy spaces of the unit disk $\Delta \subset \mathbb{C}$. Let $A$ be a Banach space of holomorphic functions on a domain $D \subset \mathbb{C}^{n}$, and assume that $A$ is contained in $L^{p}(D)$ for some $p>0$. A finite positive Borel measure $\mu$ on $D$ is a Carleson measure of $A$ if there exists a constant $C>0$ such that

$$
\forall f \in A \quad \int_{D}|f|^{p} d \mu \leq C\|f\|_{A}^{p} .
$$

Carleson studied Carleson measures of the Hardy spaces $H^{p}(\Delta)$, showing that a finite positive Borel measure $\mu$ is a Carleson measure of $H^{p}(\Delta)$ if and only if there exists a constant $C>0$ such that $\mu\left(S_{\theta_{0}, h}\right) \leq C h$ for all sets

$$
S_{\theta_{0}, h}=\left\{r e^{i \theta} \in \Delta|1-h \leq r<1,| \theta-\theta_{0} \mid \leq h\right\}
$$

(see [D]); in particular the set of Carleson measures of $H^{p}(\Delta)$ does not depend on $p$.

In 1975, Hastings $[\mathrm{H}]$ (see also Oleinik and Pavlov [OP] and Oleinik $[\mathrm{O}]$ ) proved a similar characterization for the Carleson measures of the Bergman spaces $A^{p}(\Delta)$ : a finite positive Borel measure $\mu$ is a Carleson measure of $A^{p}(\Delta)$ if and only if there exists a constant $C>0$ such that $\mu\left(S_{\theta_{0}, h}\right) \leq C h^{2}$ for all $\theta \in[0,2 \pi]$ and $h \in(0,1)$. As a consequence, again, the set of Carleson measures of $A^{p}(\Delta)$ does not depend on $p$.

The sets $S_{\theta_{0}, h}$ clearly are not invariant under automorphisms of the disk, whereas one would like to characterize Carleson measures for Bergman spaces in an invariant way, related to the intrinsic (hyperbolic) geometry of the disk and not to the extrinsic Euclidean geometry. This has been done in 1983 by Luecking [Lu]; indeed he proved (see also [DS, Theorem 14, p. 62]) that a finite positive Borel measure $\mu$ is a Carleson measure of $A^{p}(\Delta)$ if and only if for some (and hence all) $0<r<1$ there is a constant $C_{r}>0$ such that $\mu\left(B_{\Delta}\left(z_{0}, r\right)\right) \leq C_{r} \nu\left(B_{\Delta}\left(z_{0}, r\right)\right)$ for all $z_{0} \in \Delta$, where $\nu$ is the Lebesgue (area) measure and $B_{\Delta}\left(z_{0}, r\right) \subset \Delta$ is the Poincaré disk

$$
B_{\Delta}\left(z_{0}, r\right)=\left\{z \in \Delta|| \frac{z-z_{0}}{1-\overline{z_{0}} z} \mid<r\right\}
$$

of center $z_{0} \in \Delta$ and pseudohyperbolic radius $r$.

The first characterization for Carleson measures of the Bergman spaces of the unit ball $B^{n} \subset \mathbb{C}^{n}$ has been given by Cima and Wogen [CW], using again sets defined in terms of the Euclidean geometry of $B^{n}$, and thus not invariant under automorphisms. Again, as essentially noticed by Luecking [Lu] and explicitely stated by Duren and Weir [DW], it is possible to give a characterization for the Carleson measures of Bergman spaces of $B^{n}$ by using the balls for the Bergman (or Kobayashi, or pseudohyperbolic) distance: a finite positive Borel measure $\mu$ is a Carleson measure of $A^{p}\left(B^{n}\right)$ if and only if for some (and hence all) $0<r<1$ there

\footnotetext{
1 Dipartimento di Matematica, Università di Pisa, Largo Pontecorvo 5, 56127 Pisa, Italy, e-mail: abate@dm.unipi.it

2 Dipartimento di Matematica, Università di Parma, Viale G.P. Usberti 53/A, 43124 Parma, Italy, e-mail: alberto.saracco@unipr.it
} 
is a constant $C_{r}>0$ such that $\mu\left(B_{B^{n}}\left(z_{0}, r\right)\right) \leq C_{r} \nu\left(B_{B^{n}}\left(z_{0}, r\right)\right)$ for all $z_{0} \in B^{n}$, where $\nu$ is the Lebesgue $2 n$-dimensional measure and $B_{B^{n}}\left(z_{0}, r\right) \subset B^{n}$ is the ball for the Bergman distance of center $z_{0} \in B^{n}$ and radius $\frac{1}{2} \log \frac{1+r}{1-r} \in \mathbb{R}^{+}$(that is, of radius $r$ in the pseudohyperbolic distance; recall that the pseudohyperbolic distance $\rho$ is related to the Bergman or Kobayashi distance $k_{B^{n}}$ by the formula $\rho=\tanh \left(k_{B^{n}}\right)$ ).

In 1995, Cima and Mercer [CM] characterized Carleson measures of the Bergman spaces of strongly pseudoconvex domains; in particular, they proved that in this case too the class of Carleson measures of $A^{p}(D)$ does not depend on $p$. Their characterization is stated again in terms of the extrinsic Euclidean geometry of the domain, but the proof uses in an essential way the intrinsic geometry of strongly convex domains, as well as the construction of particular ad hoc functions.

A particularly important Bergman space is, of course, $A^{2}(D)$, where the Bergman kernel lives. This suggests the question of whether it is possible to characterize Carleson measures using the Bergman kernel. This has been done by Duren and Weir [DW] for the unit ball; our first main result is the generalization of their characterization to strongly pseudoconvex domains.

Let $K: D \times D \rightarrow \mathbb{C}$ be the Bergman kernel of a strongly pseudoconvex domain $D \subset \subset \mathbb{C}^{n}$. For any finite positive Borel measure $\mu$ on $D$, the Berezin transform of $\mu$ is the function $B \mu: D \rightarrow \mathbb{R}$ given by

$$
B \mu(z)=\int_{D} \frac{|K(\zeta, z)|^{2}}{K(z, z)} d \mu(\zeta) .
$$

Then (see Theorem 2.4 for a more complete statement):

Theorem 0.1: Let $\mu$ be a finite positive Borel measure on a strongly pseudoconvex bounded domain $D \subset \subset \mathbb{C}^{n}$. Then $\mu$ is a Carleson measure of $A^{p}(D)$ if and only if its Berezin transform $B \mu$ is bounded.

Previous proofs of characterizations of Carleson measures in the ball heavily relied on the homogeneity of the ball under its automorphisms group, and on the explicit expression of the automorphisms; but this approach cannot be used in our setting, because strongly pseudoconvex domains not biholomorphic to the ball admit very few automorphisms. Our proof depends instead on a detailed understanding of the intrinsic Kobayashi geometry of strongly pseudoconvex domains, and on Fefferman's estimates on the Bergman kernel.

A natural question is how to construct explicit examples of Carleson measures in strongly pseudoconvex domains. As in the unit disk and in the unit ball, an important family of examples is provided by uniformly discrete sequences. Let $(X, d)$ be a metric space; a sequence $\left\{x_{j}\right\} \subset X$ of points in $X$ is uniformly discrete if there exists $\delta>0$ such that $d\left(x_{j}, x_{k}\right) \geq \delta$ for all $j \neq k$. Then we are able to prove the following generalization of results proved by Massaneda [Ma], Jevtić, Massaneda and Thomas [JMT] and Duren and Weir [DW] in the unit ball (see Theorem 3.2 for a more complete statement):

Theorem 0.2: Let $D \subset \subset \mathbb{C}^{n}$ be a strongly pseudoconvex bounded domain, and let $\Gamma=\left\{z_{j}\right\}$ be a sequence in $D$. Then $\Gamma$ is a finite union of uniformly discrete (with respect to the Kobayashi distance) sequences if and only if $\sum_{z_{j} \in \Gamma} d\left(z_{j}, \partial D\right)^{n+1} \delta_{z_{j}}$ is a Carleson measure of $A^{p}(D)$, where $\delta_{z_{j}}$ is the Dirac measure at $z_{j}$ and $d(\cdot, \partial D)$ is the Euclidean distance from the boundary.

Finally, Duren, Schuster and Vukotić [DSV], Duren and Weir [DW], and MacCluer [Mc] also studied how fast in the unit ball a uniformly discrete (with respect to the pseudohyperbolic or Bergman distances of the ball) sequence escapes to the boundary. We are able to generalize their results to strongly pseudoconvex domains (see Theorem 3.6, and Proposition 3.4 for a similar result not requiring pseudoconvexity):

Theorem 0.3: Let $D \subset \mathbb{C}^{n}$ be a strongly pseudoconvex bounded domain. Let $\Gamma=\left\{z_{j}\right\} \subset D$ be an uniformly discrete (with respect to the Kobayashi distance) sequence with $d\left(z_{j}, \partial D\right)<1$ for all $j \in \mathbb{N}^{*}$. Then

$$
\sum_{z_{j} \in \Gamma} d\left(z_{j}, \partial D\right)^{n} h\left(-\frac{1}{\log d\left(z_{j}, \partial D\right)}\right)<+\infty
$$

for all increasing functions $h: \mathbb{R}^{+} \rightarrow \mathbb{R}^{+}$such that

$$
\sum_{m=1}^{+\infty} h\left(\frac{1}{m}\right)<+\infty
$$




\section{The intrinsic geometry of strongly convex domains}

In this section we shall prove a number of estimates on the intrinsic geometry of strongly convex domains, as described by the Kobayashi distance. In particular, we shall study the boundary behavior of Kobayashi balls, and we shall prove a sort of submean property for nonnegative plurisubharmonic functions in Kobayashi balls.

Let us briefly recall the definition and the main properties of the Kobayashi distance; we refer to [A], $[\mathrm{JP}]$ and $[\mathrm{K}]$ for details and much more. Let $k_{\Delta}$ denote the Poincaré distance on the unit disk $\Delta \subset \mathbb{C}$. If $X$ is a complex manifold, the Lempert function $\delta_{X}: X \times X \rightarrow \mathbb{R}^{+}$of $X$ is defined by

$$
\delta_{X}(z, w)=\inf \left\{k_{\Delta}(\zeta, \eta) \mid \text { there exists a holomorphic } \phi: \Delta \rightarrow X \text { with } \phi(\zeta)=z \text { and } \phi(\eta)=w\right\}
$$

for all $z, w \in X$. The Kobayashi pseudodistance $k_{X}: X \times X \rightarrow \mathbb{R}^{+}$of $X$ is the smallest pseudodistance on $X$ bounded below by $\delta_{X}$. We say that $X$ is (Kobayashi) hyperbolic if $k_{X}$ is a true distance - and in that case it is known that the metric topology induced by $k_{X}$ coincides with the manifold topology of $X$ (see, e.g., [A, Proposition 2.3.10]). For instance, all bounded domains are hyperbolic (see, e.g., [A, Theorem 2.3.14]).

The main property of the Kobayashi (pseudo)distance is that it is contracted by holomorphic maps: if $f: X \rightarrow Y$ is a holomorphic map then

$$
\forall z, w \in X \quad k_{Y}(f(z), f(w)) \leq k_{X}(z, w) .
$$

In particular, the Kobayashi distance is invariant under biholomorphisms, and decreases under inclusions: if $D_{1} \subset D_{2} \subset \subset \mathbb{C}^{n}$ are two bounded domains we have $k_{D_{2}}(z, w) \leq k_{D_{1}}(z, w)$ for all $z, w \in D_{1}$.

It is easy to see that the Kobayashi distance of the unit disk coincides with the Poincare distance. Furthermore, the Kobayashi distance of the unit ball $B^{n} \subset \mathbb{C}^{n}$ coincides with the Bergman distance (see, e.g., [A, Corollary 2.3.6]).

If $X$ is a hyperbolic manifold, $z_{0} \in X$ and $r \in(0,1)$ we shall denote by $B_{X}\left(z_{0}, r\right)$ the Kobayashi ball of center $z_{0}$ and radius $\frac{1}{2} \log \frac{1+r}{1-r}$ :

$$
B_{X}\left(z_{0}, r\right)=\left\{z \in X \mid \tanh k_{X}\left(z_{0}, z\right)<r\right\} .
$$

Notice that $\rho_{X}=\tanh k_{X}$ is still a distance on $X$, because tanh is a strictly convex function on $\mathbb{R}^{+}$. In particular, $\rho_{B^{n}}$ is the pseudohyperbolic distance of $B^{n}$.

The Kobayashi distance of bounded strongly pseudoconvex domains enjoys several important properties. First of all, it is complete (see, e.g., [A, Corollary 2.3.53]), and hence closed Kobayashi balls are compact. Furthermore, we can very precisely describe the boundary behavior of the Kobayashi distance: if $D \subset \subset \mathbb{C}^{n}$ is a strongly pseudoconvex bounded domain and $z_{0} \in D$, there exist $c_{0}, C_{0}>0$ such that

$$
\forall z \in D \quad c_{0}-\frac{1}{2} \log d(z, \partial D) \leq k_{D}\left(z_{0}, z\right) \leq C_{0}-\frac{1}{2} \log d(z, \partial D),
$$

where $d(\cdot, \partial D)$ denotes the Euclidean distance from the boundary of $D$ (see [A, Theorems 2.3.51 and 2.3.52]).

Let us finally recall a couple of facts on Kobayashi balls of $B^{n}$; for proofs see [A, Section 2.2.2], [Ru, Section 2.2.7] and [DW]. The ball $B_{B^{n}}\left(z_{0}, r\right)$ is given by

$$
B_{B^{n}}\left(z_{0}, r\right)=\left\{z \in B^{n} \mid \frac{\left(1-\left\|z_{0}\right\|^{2}\right)\left(1-\|z\|^{2}\right)}{\left|1-\left\langle z, z_{0}\right\rangle\right|^{2}}>1-r^{2}\right\}
$$

Geometrically, it is an ellipsoid of (Euclidean) center

$$
c=\frac{1-r^{2}}{1-r^{2}\left\|z_{0}\right\|^{2}} z_{0},
$$

its intersection with the complex line $\mathbb{C} z_{0}$ is an Euclidean disk of radius

$$
r \frac{1-\left\|z_{0}\right\|^{2}}{1-r^{2}\left\|z_{0}\right\|^{2}},
$$


and its intersection with the affine subspace through $z_{0}$ orthogonal to $z_{0}$ is an Euclidean ball of the larger radius

$$
r \sqrt{\frac{1-\left\|z_{0}\right\|^{2}}{1-r^{2}\left\|z_{0}\right\|^{2}}}
$$

Let $\nu$ denote the Lebesgue volume measure of $\mathbb{R}^{2 n}$, normalized so that $\nu\left(B^{n}\right)=1$. Then the volume of a Kobayashi ball $B_{B^{n}}\left(z_{0}, r\right)$ is given by (see $\left.[\mathrm{DW}]\right)$

$$
\nu\left(B_{B^{n}}\left(z_{0}, r\right)\right)=r^{2 n}\left(\frac{1-\left\|z_{0}\right\|^{2}}{1-r^{2}\left\|z_{0}\right\|^{2}}\right)^{n+1}
$$

A similar estimate holds for the volume of Kobayashi balls in strongly pseudoconvex bounded domains:

Lemma 1.1: Let $D \subset \subset \mathbb{C}^{n}$ be a strongly pseudoconvex bounded domain. Then there exist $c_{1}>0$ and, for each $r \in(0,1)$, a $C_{1, r}>0$ depending on $r$ such that

$$
c_{1} r^{2 n} d\left(z_{0}, \partial D\right)^{n+1} \leq \nu\left(B_{D}\left(z_{0}, r\right)\right) \leq C_{1, r} d\left(z_{0}, \partial D\right)^{n+1}
$$

for every $z_{0} \in D$ and $r \in(0,1)$.

Proof: Since $D$ is bounded and with smooth boundary, there is $\delta>0$ so that any euclidean ball internally tangent to $\partial D$ with radius $\delta$ is completely contained in $D$. Take $z_{0} \in D$ with $d\left(z_{0}, \partial D\right)<\delta$, and choose $x \in \partial D$ so that $d\left(z_{0}, \partial D\right)=\left\|x-z_{0}\right\|$. Then the euclidean ball $B$ tangent to $\partial D$ in $x$ and with radius $\delta$ is contained in $D$, contains $z_{0}$ and $d\left(z_{0}, \partial D\right)=d\left(z_{0}, \partial B\right)$. Since the Kobayashi distance decreases under inclusions, $B_{B}\left(z_{0}, r\right) \subset B_{D}\left(z_{0}, r\right)$. Hence (1.3) yields (assuming without loss of generality that $B$ is centered at the origin)

$$
\begin{aligned}
\nu\left(B_{D}\left(z_{0}, r\right)\right) & \geq \nu\left(B_{B}\left(z_{0}, r\right)\right)=\delta^{2 n} \nu\left(B_{B^{n}}\left(z_{0} / \delta, r\right)\right) \\
& =\delta^{2 n} r^{2 n}\left(\frac{1-\left\|z_{0} / \delta\right\|^{2}}{1-r^{2}\left\|z_{0} / \delta\right\|^{2}}\right)^{n+1}=\delta^{2 n} r^{2 n}\left(\frac{\delta^{2}-\left\|z_{0}\right\|^{2}}{\delta^{2}-r^{2}\left\|z_{0}\right\|^{2}}\right)^{n+1} \\
& \geq \delta^{2 n} r^{2 n}\left(\frac{\delta\left(\delta-\left\|z_{0}\right\|\right)}{\delta^{2}}\right)^{n+1}=\delta^{n-1} r^{2 n} d\left(z_{0}, \partial D\right)^{n+1}
\end{aligned}
$$

and we are done in this case.

Assume now $d\left(z_{0}, \partial D\right) \geq \delta$, and let $B \subset D$ the euclidean ball of center $z_{0}$ and radius $d\left(z_{0}, \partial D\right)$. Then $B_{B}\left(z_{0}, r\right)$ is an euclidean ball of radius $r d\left(z_{0}, \partial D\right)$, and so

$$
\nu\left(B_{D}\left(z_{0}, r\right)\right) \geq \nu\left(B_{B}\left(z_{0}, r\right)\right)=r^{2 n} d\left(z_{0}, \partial D\right)^{2 n} \geq \delta^{n-1} r^{2 n} d\left(z_{0}, \partial D\right)^{n+1},
$$

and we have obtained the lower estimate in this case too.

For the upper estimate, results of $[\mathrm{KM}]$ and $[\mathrm{Li}]$ show that there is a $\delta_{1}>0$ such that if $d\left(z_{0}, \partial D\right)<\delta_{1}$ then there exist $a_{1}(r), a_{2}(r)>0$ so that $B_{D}\left(z_{0}, r\right)$ is contained in a (possibly rotated) polydisk $P$ of center $z_{0}$ and polyradius $\left(a_{1}(r) d\left(z_{0}, \partial D\right), a_{2}(r) \sqrt{d\left(z_{0}, \partial D\right)}, \ldots, a_{2}(r) \sqrt{d\left(z_{0}, \partial D\right)}\right)$. Thus if $d\left(z_{0}, \partial D\right)<\delta_{1}$ we have

$$
\nu\left(B_{D}\left(z_{0}, r\right)\right) \leq \nu(P) \leq c a_{1}(r)^{2} a_{2}(r)^{2(n-1)} d\left(z_{0}, \partial D\right)^{n+1}
$$

for a suitable constant $c>0$ independent of $r$.

Finally, if $d\left(z_{0}, \partial D\right) \geq \delta_{1}$ we have

$$
\nu\left(B_{D}\left(z_{0}, r\right)\right) \leq \frac{\nu(D)}{\delta_{1}^{n+1}} d\left(z_{0}, \partial D\right)^{n+1}
$$

and we are done. 
Remark 1.1: If $D$ is strongly convex, using Euclidean balls externally tangent to $D$ it is possible to show that one can take

$$
C_{1, r}=C_{1} r^{2 n}\left(1-r^{2}\right)^{-(n+1)},
$$

where $C_{1}>0$ is a constant independent of $r$.

The next lemma contains an estimate on the Euclidean size of Kobayashi balls.

Lemma 1.2: Let $D \subset \subset \mathbb{C}^{n}$ be a strongly pseudoconvex bounded domain. Then there is $C_{2}>0$ such that for every $z_{0} \in D$ and $r \in(0,1)$ one has

$$
\forall z \in B_{D}\left(z_{0}, r\right) \quad \frac{C_{2}}{1-r} d\left(z_{0}, \partial D\right) \geq d(z, \partial D) \geq \frac{1-r}{C_{2}} d\left(z_{0}, \partial D\right) .
$$

Proof: Let us fix $w_{0} \in D$. Then (1.1) yields $c_{0}, C_{0}>0$ such that

$$
c_{0}-\frac{1}{2} \log d(z, \partial D) \leq k_{D}\left(w_{0}, z\right) \leq k_{D}\left(z_{0}, z\right)+k_{D}\left(z_{0}, w_{0}\right) \leq \frac{1}{2} \log \frac{1+r}{1-r}+C_{0}-\frac{1}{2} \log d\left(z_{0}, \partial D\right),
$$

for all $z \in B_{D}\left(z_{0}, r\right)$, and hence

$$
e^{2\left(c_{0}-C_{0}\right)} d\left(z_{0}, \partial D\right) \leq \frac{2}{1-r} d(z, \partial D) .
$$

The left-hand inequality is obtained in the same way reversing the roles of $z_{0}$ and $z$.

Remark 1.2: Using again externally tangent Euclidean balls it is possible to show that if $D$ is strongly convex then one can take $C_{2}=4$.

The two previous lemmas (together with the following Corollary 1.7) give the main properties needed in Luecking's approach $[\mathrm{Lu}]$ to the characterization of Carleson measures. However, to get the characterization involving the Berezin transform, we shall need precise information on the behaviour of the Bergman kernel in Kobayashi balls, that we shall deduce from Fefferman's estimates using another estimate on the shape of Kobayashi balls. In the unit ball the latter estimate has the following form:

Lemma 1.3: Let $B^{n} \subset \mathbb{C}^{n}$ be the unit ball in $\mathbb{C}^{n}$, and take $z_{0} \in B^{n}$ and $r \in(0,1)$. Then

$$
\forall z \in B_{B^{n}}\left(z_{0}, r\right) \quad 1-\left\|z_{0}\right\|^{2}>\frac{1-r^{2}}{4}\left(\left\|z-z_{0}\right\|^{2}+\left|\left\langle z-z_{0}, z_{0}\right\rangle\right|\right) .
$$

Proof: First of all, it is easy to check that $z \in B_{B^{n}}\left(z_{0}, r\right)$ if and only if

$$
\frac{\left(1-\left\|z_{0}\right\|^{2}\right)\left(1-\|z\|^{2}\right)}{\left|1-\left\langle z, z_{0}\right\rangle\right|^{2}}>1-r^{2}
$$

therefore it suffices to prove that

$$
\frac{\left|1-\left\langle z, z_{0}\right\rangle\right|^{2}}{1-\|z\|^{2}} \geq \frac{1}{4}\left(\left\|z-z_{0}\right\|^{2}+\left|\left\langle z-z_{0}, z_{0}\right\rangle\right|\right) .
$$

Let us write $z_{0}=\lambda z+w$, with $\lambda \in \mathbb{C}$ and $w \perp z$. Then $\left\langle z, z_{0}\right\rangle=\bar{\lambda}\|z\|^{2}$ and $\left\|z-z_{0}\right\|^{2}=|1-\lambda|^{2}\|z\|^{2}+\|w\|^{2}$; moreover $|\lambda|^{2}\|z\|^{2}+\|w\|^{2}=\left\|z_{0}\right\|^{2}<1$, that is $\|w\|^{2}<1-|\lambda|^{2}\|z\|^{2}$. Now, a computation shows that $\|z\|^{4}|\lambda-1|^{2} \geq 0$ is equivalent to

$$
\left|1-\bar{\lambda}\|z\|^{2}\right|^{2} \geq\left(1-\|z\|^{2}\right)\left(|1-\lambda|^{2}\|z\|^{2}+1-|\lambda|^{2}\|z\|^{2}\right) ;
$$

therefore

$$
\frac{\left|1-\left\langle z, z_{0}\right\rangle\right|^{2}}{1-\|z\|^{2}}=\frac{\left|1-\bar{\lambda}\|z\|^{2}\right|^{2}}{1-\|z\|^{2}} \geq|1-\lambda|^{2}\|z\|^{2}+1-|\lambda|^{2}\|z\|^{2}>|1-\lambda|^{2}\|z\|^{2}+\|w\|^{2}=\left\|z-z_{0}\right\|^{2} .
$$


Write $z=\mu z_{0}+w$, with $\mu \in \mathbb{C}$ and $w \perp z_{0}$. Then $\left\langle z, z_{0}\right\rangle=\mu\left\|z_{0}\right\|^{2}$ and $\left|\left\langle z-z_{0}, z_{0}\right\rangle\right|=|1-\mu|\left\|z_{0}\right\|^{2}$; moreover $|\mu|^{2}\left\|z_{0}\right\|^{2}+\|w\|^{2}=\|z\|^{2}<1$, implying $|\mu|\left\|z_{0}\right\|<1$. Now,

$$
\frac{\left|1-\left\langle z, z_{0}\right\rangle\right|^{2}}{1-\|z\|^{2}}=\frac{\left|1-\mu\left\|z_{0}\right\|^{2}\right|^{2}}{1-|\mu|^{2}\left\|z_{0}\right\|^{2}-\|w\|^{2}} \geq \frac{\left|1-\mu\left\|z_{0}\right\|^{2}\right|^{2}}{1-|\mu|^{2}\left\|z_{0}\right\|^{2}}=\frac{\left|1-\mu\left\|z_{0}\right\|^{2}\right|}{1-|\mu|\left\|z_{0}\right\|} \cdot \frac{\left|1-\mu\left\|z_{0}\right\|^{2}\right|}{1+|\mu|\left\|z_{0}\right\|} .
$$

By assumption we have $2 \geq|\mu|\left\|z_{0}\right\|\left(1+\left\|z_{0}\right\|\right)$. A computation shows that this is equivalent to

$$
|1-| \mu||\left|z_{0} \|^{2}\right|^{2} \geq\left(1-|\mu||| z_{0} \|\right)^{2} ;
$$

therefore

$$
\frac{|1-\mu|\left|z_{0} \|^{2}\right|}{1-|\mu|\left\|z_{0}\right\|} \geq \frac{|1-| \mu||\left|z_{0} \|^{2}\right|}{1-|\mu|\left\|z_{0}\right\|} \geq 1
$$

Next, $1+\left\|z_{0}\right\|^{2} \geq 2\left\|z_{0}\right\| \geq 2|\mu|\left\|z_{0}\right\|^{2} \geq 2\left\|z_{0}\right\|^{2} \operatorname{Re} \mu$; multiplying this by $1-\left\|z_{0}\right\|^{2}$ we end up with

$$
\left|1-\mu\left\|z_{0}\right\|^{2}\right|^{2} \geq|1-\mu|^{2}\left\|z_{0}\right\|^{4}=\left|\left\langle z-z_{0}, z_{0}\right\rangle\right|^{2} .
$$

Since $1+|\mu|\left\|z_{0}\right\|<2$, putting together (1.6), (1.7) and (1.8) we get

$$
\frac{\left|1-\left\langle z, z_{0}\right\rangle\right|^{2}}{1-\|z\|^{2}}>\frac{1}{2}\left|\left\langle z-z_{0}, z_{0}\right\rangle\right| .
$$

Putting together (1.5) and (1.9) we get (1.4), and thus the assertion.

A defining function for a smooth domain $D \subset \subset \mathbb{C}^{n}$ is a smooth function $\psi: \mathbb{C}^{n} \rightarrow \mathbb{R}$ such that $D=\{\psi>0\}$ and the gradient $\nabla \psi$ is never zero on $\partial D$. If $D$ is strongly pseudoconvex then we can find a defining function strictly plurisubharmonic in a neighbourhood of $\partial D$.

Lemma 1.4: Let $D \subset \subset \mathbb{C}^{n}$ be a strongly pseudoconvex bounded domain, and $\psi: \mathbb{C}^{n} \rightarrow \mathbb{R}$ a defining function for $D$. Then for every $r \in(0,1)$ there exists $c_{2, r}>0$ depending on $r$ such that for every $z_{0} \in D$ one has

$$
\forall z \in B_{D}\left(z_{0}, r\right) \quad d\left(z_{0}, \partial D\right) \geq c_{2, r}\left(\left\|z-z_{0}\right\|^{2}+\left|\partial \psi_{z_{0}}\left(z-z_{0}\right)\right|\right) .
$$

Proof: Fix $r \in(0,1)$. Since $D$ is bounded, it suffices to prove the statement for $z_{0}$ close enough to $\partial D$.

By Narasimhan's lemma [Kr, Lemma 3.2.3] we can cover $\partial D$ with open sets $U_{1}, \ldots, U_{l} \subset \mathbb{C}^{n}$ so that for each $j=1, \ldots, l$ there is a biholomorphism $\Phi_{j}: U_{j} \rightarrow \Phi_{j}\left(U_{j}\right) \subset \mathbb{C}^{n}$ such that $\Phi_{j}\left(U_{j} \cap D\right)$ is strongly convex. Furthermore, we can assume that each $\Phi_{j}$ is defined in a slightly larger neighborhood, and hence find $c>0$ such that $d\left(z_{0}, \partial D\right) \geq c d\left(\Phi_{j}\left(z_{0}\right), \partial \Phi_{j}\left(U_{j} \cap D\right)\right)$ for all $z_{0} \in U_{j} \cap D$ close enough to $\partial D$ and all $j=1, \ldots, l$. For the same reason, and recalling Lemma 1.2, $\left|\partial \psi_{z_{0}}\left(z-z_{0}\right)\right|$ and $\left|\partial\left(\psi \circ \Phi_{j}^{-1}\right)_{\Phi_{j}\left(z_{0}\right)}\left(\Phi_{j}(z)-\Phi_{j}\left(z_{0}\right)\right)\right|$ are comparable as soon as $z_{0} \in U_{j} \cap D$ is close enough to $\partial D$. Finally, by the localization property of the Kobayashi distance (see [A, Theorem 2.3.65]) if $z_{0} \in U_{j} \cap D$ is close enough to the boundary of $D$ we can find $r_{1} \in(0,1)$ depending only on $r$ such that $B_{D}\left(z_{0}, r\right) \subseteq B_{U_{j} \cap D}\left(z_{0}, r_{1}\right)$. The upshot of these remarks is that it suffices to prove the statement when $D$ is strongly convex.

So, assume $D$ strongly convex, and let $\delta>0$ be such that if $d\left(z_{0}, \partial D\right)<\delta$ then there exists a unique $x=x\left(z_{0}\right) \in \partial D$ so that $d\left(z_{0}, \partial D\right)=\left\|x-z_{0}\right\|$; again it suffices to prove the statement for $d\left(z_{0}, \partial D\right)<\delta$.

Take then $z_{0} \in D$ with $d\left(z_{0}, \partial D\right)<\delta$; since $D$ is strongly convex, there exists an euclidean ball $B$ containing $D$ and tangent to $D$ in $x=x\left(z_{0}\right)$; in particular, $d\left(z_{0}, \partial D\right)=d\left(z_{0}, \partial B\right)$. Let $R=R\left(z_{0}\right)>0$ be the radius of $B$; up to a translation, we can assume that $B$ is centered at the origin.

Let $z \in B_{D}\left(z_{0}, r\right)$. Since $B_{D}\left(z_{0}, r\right) \subseteq B_{B}\left(z_{0}, r\right)$, Lemma 1.3 implies

$$
2 R d\left(z_{0}, \partial D\right) \geq R^{2}-\left\|z_{0}\right\|^{2}>\frac{1-r^{2}}{4}\left(\left\|z-z_{0}\right\|^{2}+\left|\left\langle z-z_{0}, z_{0}\right\rangle\right|\right) .
$$

Now, $\left|\left\langle z-z_{0}, z_{0}\right\rangle\right|$ is (a uniform multiple of) the distance of $z$ from the complex hyperplane $\pi$ passing through $z_{0}$ and parallel to the complex hyperplane tangent to $\partial B$ at $x$. But the latter coincides with the complex hyperplane tangent to $\partial D$ at $x$, which is exactly given by $\partial \psi_{x}(z-x)=0$. Therefore $\pi$ has equation $\partial \psi_{x}\left(z-z_{0}\right)=0$. It follows that the difference between $\left|\left\langle z-z_{0}, z_{0}\right\rangle\right|$ and $\left|\partial \psi_{z_{0}}\left(z-z_{0}\right)\right|$ is (uniformly in $z$ and $\left.z_{0}\right)$ of the order of $\left\|z-z_{0}\right\|\left\|z_{0}-x\right\|=d\left(z_{0}, \partial D\right)\left\|z-z_{0}\right\| ;$ so we get

$$
\left(2 R+C\left\|z-z_{0}\right\|\left(1-r^{2}\right)\right) d\left(z_{0}, \partial D\right) \geq \frac{1-r^{2}}{4}\left(\left\|z-z_{0}\right\|^{2}+\left|\partial \psi_{z_{0}}\left(z-z_{0}\right)\right|\right)
$$

for a suitable constant $C>0$. Since $D$ is bounded, $R$ too is bounded as a function of $z_{0}$, and the assertion follows. 
Remark 1.3: If $D$ is strongly convex the proof shows that we can take $c_{2, r}=c_{2}\left(1-r^{2}\right)$ for a suitable $c_{2}>0$ independent of $r$.

We now prove a covering lemma for $D$.

Lemma 1.5: Let $D \subset \subset \mathbb{C}^{n}$ be a strongly pseudoconvex bounded domain. Then for every $r \in(0,1)$ there exist $m \in \mathbb{N}$ and a sequence $\left\{z_{k}\right\} \subset D$ of points such that $D=\bigcup_{k=0}^{\infty} B\left(z_{k}, r\right)$ and no point of $D$ belongs to more than $m$ of the balls $B_{D}\left(z_{k}, R\right)$, where $R=\frac{1}{2}(1+r)$.

Proof: Let $\left\{B_{j}\right\}_{j \in \mathbb{N}}$ be a sequence of Kobayashi balls of radius $r / 3$ covering $D$. We can extract a subsequence $\left\{\Delta_{k}=B_{D}\left(z_{k}, r / 3\right)\right\}_{k \in \mathbb{N}}$ of disjoint balls in the following way: set $\Delta_{1}=B_{1}$. Suppose we have already chosen $\Delta_{1}, \ldots, \Delta_{l}$. We define $\Delta_{l+1}$ as the first ball in the sequence $\left\{B_{j}\right\}$ which is disjoint from $\Delta_{1} \cup \cdots \cup \Delta_{l}$. In particular, by construction every $B_{j}$ must intersect at least one $\Delta_{k}$.

We now claim that $\left\{B_{D}\left(z_{k}, r\right)\right\}_{k \in \mathbb{N}}$ is a covering of $D$. Indeed, let $z \in D$. Since $\left\{B_{j}\right\}_{j \in \mathbb{N}}$ is a covering of $D$, there is $j_{0} \in \mathbb{N}$ so that $z \in B_{j_{0}}$. As remarked above, we get $k_{0} \in \mathbb{N}$ so that $B_{j_{0}} \cap \Delta_{k_{0}} \neq \varnothing$. Take $w \in B_{j_{0}} \cap \Delta_{k_{0}}$. Then

$$
\rho_{D}\left(z, z_{k_{0}}\right) \leq \rho_{D}(z, w)+\rho_{D}\left(w, z_{k_{0}}\right) \leq \frac{2}{3} r
$$

and $z \in B_{D}\left(z_{k_{0}}, r\right)$.

To conclude the proof we have to show that there is $m=m_{r} \in \mathbb{N}$ so that each point $z \in D$ belongs to at most $m$ of the balls $B\left(z_{k}, R\right)$. Put $r_{1}=\frac{1}{3} \min \{r, 1-r\}$ and $R_{1}=\frac{1}{6}(5+r)$. Since $z \in B_{D}\left(z_{k}, R\right)$ is equivalent to $z_{k} \in B_{D}(z, R)$, we have that $z \in B_{D}\left(z_{k}, R\right)$ implies $B_{D}\left(z_{k}, r_{1}\right) \subset B_{D}\left(z, R_{1}\right)$. Furthermore, Lemmas 1.1 and 1.2 yields

$$
\nu\left(B_{D}\left(z_{k}, r_{1}\right)\right) \geq c_{1} r_{1}^{2 n} d\left(z_{k}, \partial D\right)^{n+1} \geq \frac{c_{1}}{C_{2}^{n+1}}(1-R)^{n+1} r_{1}^{2 n} d(z, \partial D)^{n+1}
$$

when $z_{k} \in B_{D}(z, R)$. Therefore, since the balls $B_{D}\left(z_{k}, r_{1}\right)$ are pairwise disjoint, using again Lemma 1.1 we get

$$
\operatorname{card}\left\{k \in \mathbb{N} \mid z \in B_{D}\left(z_{k}, R\right)\right\} \leq \frac{\nu\left(B_{D}\left(z, R_{1}\right)\right)}{\nu\left(B_{D}\left(z_{k}, r_{1}\right)\right)} \leq \frac{C_{2}^{n+1} C_{1, R_{1}}}{c_{1}} \frac{1}{r_{1}^{2 n}(1-R)^{n+1}},
$$

and we are done.

Our last aim for this section is a sort of submean property in Kobayashi balls for nonnegative plurisubharmonic functions. Let us first prove it in an Euclidean ball:

Lemma 1.6: Let $B \subset \subset \mathbb{C}^{N}$ be an Euclidean ball of radius $R>0$. Then

$$
\forall z_{0} \in B \forall r \in(0,1) \quad \chi\left(z_{0}\right) \leq \frac{4^{n+1}}{R^{n-1}} \frac{1}{r^{2 n} d\left(z_{0}, \partial B\right)^{n+1}} \int_{B_{B}\left(z_{0}, r\right)} \chi d \nu
$$

for all nonnegative plurisubharmonic functions $\chi: B \rightarrow \mathbb{R}^{+}$.

Proof: Without loss of generality we can assume that $B$ is centered at the origin. Let $\phi_{z_{0} / R}: B^{n} \rightarrow B^{n}$ be the usual involutive automorphism of $B^{n}$ sending the origin in $z_{0} / R$ (see [Ru, Section 2.2]), and let $\Phi_{z_{0}}: B^{n} \rightarrow B$ be given by $\Phi_{z_{0}}=R \phi_{z_{0} / R}$; in particular, $\Phi_{z_{0}}$ is a biholomorphism with $\Phi_{z_{0}}(O)=z_{0}$, and thus $\Phi_{z_{0}}\left(B_{B^{n}}(O, r)\right)=B_{B}\left(z_{0}, r\right)$. Furthermore (see $[\mathrm{Ru}$, Theorem 2.2.6])

$$
\left|\operatorname{Jac}_{\mathbb{R}} \Phi_{z_{0}}(z)\right|=R^{2 n}\left(\frac{R^{2}-\left\|z_{0}\right\|^{2}}{\left|R-\left\langle z, z_{0}\right\rangle\right|^{2}}\right)^{n+1} \geq \frac{R^{n-1}}{4^{n+1}} d\left(z_{0}, \partial B\right)^{n+1},
$$

where $\operatorname{Jac}_{\mathbb{R}} \Phi_{z_{0}}$ denotes the (real) Jacobian determinant of $\Phi_{z_{0}}$. It follows that

$$
\int_{B\left(z_{0}, r\right)} \chi d \nu=\int_{B_{B^{n}}(O, r)}\left(\chi \circ \Phi_{z_{0}}\right)\left|\operatorname{Jac}_{\mathbb{R}} \Phi_{z_{0}}\right| d \nu \geq \frac{R^{n-1}}{4^{n+1}} d\left(z_{0}, \partial B\right)^{n+1} \int_{B_{B^{n}(O, r)}}\left(\chi \circ \Phi_{z_{0}}\right) d \nu .
$$

Using [Ru, 1.4.3 and 1.4.7.(1)] we obtain

$$
\int_{B_{B^{n}}(O, r)}\left(\chi \circ \Phi_{z_{0}}\right) d \nu=2 n \int_{\partial B^{n}} d \sigma(x) \frac{1}{2 \pi} \int_{0}^{r} \int_{0}^{2 \pi} \chi \circ \Phi_{z_{0}}\left(t e^{i \theta} x\right) t^{2 n-1} d t d \theta,
$$


where $\sigma$ is the area measure on $\partial B^{n}$ normalized so that $\sigma\left(\partial B^{n}\right)=1$. Now, $\zeta \mapsto \chi \circ \Phi_{z_{0}}(\zeta x)$ is subharmonic on $r \Delta=\{|\zeta|<r\} \subset \mathbb{C}$ for any $x \in \partial B^{n}$. Therefore [Hö, Theorem 1.6.3] yields

$$
\frac{1}{2 \pi} \int_{0}^{r} \int_{0}^{2 \pi} \chi \circ \Phi_{z_{0}}\left(t e^{i \theta} x\right) t^{2 n-1} d t d \theta \geq \chi\left(z_{0}\right) \int_{0}^{r} t^{2 n-1} d t=\frac{1}{2 n} r^{2 n} \chi\left(z_{0}\right) .
$$

So

$$
\int_{B_{B^{n}}(O, r)}\left(\chi \circ \Phi_{z_{0}}\right) d \nu \geq r^{2 n} \chi\left(z_{0}\right)
$$

and the assertion follows.

Then:

Corollary 1.7: Let $D \subset \subset \mathbb{C}^{n}$ be a strongly pseudoconvex bounded domain, and $r \in(0,1)$. Then there exists a $C_{3, r}>0$ depending on $r$ such that

$$
\forall z_{0} \in D \quad \chi\left(z_{0}\right) \leq \frac{C_{3, r}}{\nu\left(B_{D}\left(z_{0}, r\right)\right)} \int_{B_{D}\left(z_{0}, r\right)} \chi d \nu
$$

for all non-negative plurisubharmonic functions $\chi: D \rightarrow \mathbb{R}^{+}$.

Proof: Since $D$ has smooth boundary, there exists a radius $\rho>0$ such that for every $x \in \partial D$ the euclidean ball $B_{x}(\rho)$ internally tangent to $\partial D$ at $x$ is contained in $D$.

Let $z_{0} \in D$. If $d\left(z_{0}, \partial D\right) \leq \rho$, let $x \in \partial D$ be such that $d\left(z_{0}, \partial D\right)=\left\|z_{0}-x\right\|$; in particular, $z_{0}$ belongs to the ball $B=B_{x}(\rho) \subset D$. If $d\left(z_{0}, \partial D\right)>\rho$, let $B \subset D$ be the Euclidean ball of center $z_{0}$ and radius $d\left(z_{0}, \partial D\right)$. In both cases we have $d\left(z_{0}, \partial D\right)=d\left(z_{0}, \partial B\right)$; moreover, the decreasing property of the Kobayashi distance yields $B_{D}\left(z_{0}, r\right) \supseteq B_{B}\left(z_{0}, r\right)$ for all $r \in(0,1)$.

Let $\chi$ be a non-negative plurisubharmonic function. Then Lemmas 1.6 and 1.1 imply

$$
\begin{aligned}
\int_{B_{D}\left(z_{0}, r\right)} \chi d \nu & \geq \int_{B_{B}\left(z_{0}, r\right)} \chi d \nu \geq \frac{\rho^{n-1}}{4^{n+1}} \frac{r^{2 n} d\left(z_{0}, \partial D\right)^{n+1}}{\nu\left(B_{D}\left(z_{0}, r\right)\right)} \nu\left(B_{D}\left(z_{0}, r\right)\right) \chi\left(z_{0}\right) \\
& \geq \frac{\rho^{n-1}}{4^{n+1} C_{1, r}} \nu\left(B_{D}\left(z_{0}, r\right)\right) \chi\left(z_{0}\right)
\end{aligned}
$$

and we are done.

Remark 1.4: If $D$ is strongly convex then one can take $C_{3, r}=C_{3}\left(1-r^{2}\right)^{-(n+1)}$, where $C_{3}>0$ is independent of $r$.

In a similar way we get another useful estimate:

Corollary 1.8: Let $D \subset \subset \mathbb{C}^{n}$ be a strongly pseudoconvex bounded domain. Given $r \in(0,1)$, set $R=\frac{1}{2}(1+r) \in(0,1)$. Then there exists a $K_{r}>0$ depending on $r$ such that

$$
\forall z_{0} \in D \forall z \in B_{D}\left(z_{0}, r\right) \quad \chi(z) \leq \frac{K_{r}}{\nu\left(B_{D}\left(z_{0}, r\right)\right)} \int_{B_{D}\left(z_{0}, R\right)} \chi d \nu
$$

for every nonnegative plurisubharmonic function $\chi: D \rightarrow \mathbb{R}^{+}$.

Proof: Let $r_{1}=\frac{1}{2}(1-r)$; the triangle inequality implies that $z \in B_{D}\left(z_{0}, r\right)$ yields $B_{D}\left(z, r_{1}\right) \subset B_{D}\left(z_{0}, R\right)$. Corollary 1.7 then implies

$$
\begin{aligned}
\chi(z) & \leq \frac{C_{3, r_{1}}}{\nu\left(B_{D}\left(z, r_{1}\right)\right)} \int_{B_{D}\left(z, r_{1}\right)} \chi d \nu \leq \frac{C_{3, r_{1}}}{\nu\left(B_{D}\left(z, r_{1}\right)\right)} \int_{B_{D}\left(z_{0}, R\right)} \chi d \nu \\
& =C_{3, r_{1}} \frac{\nu\left(B_{D}\left(z_{0}, r\right)\right)}{\nu\left(B_{D}\left(z, r_{1}\right)\right)} \cdot \frac{1}{\nu\left(B_{D}\left(z_{0}, r\right)\right)} \int_{B_{D}\left(z_{0}, R\right)} \chi d \nu
\end{aligned}
$$


for all $z \in B_{D}\left(z_{0}, r\right)$. Now Lemmas 1.2 and 1.1 yield

$$
\frac{\nu\left(B_{D}\left(z_{0}, r\right)\right)}{\nu\left(B_{D}\left(z, r_{1}\right)\right)} \leq \frac{C_{1, r} C_{2}^{n+1}}{c_{1}(1-r)^{n+1} r_{1}^{2 n}}
$$

and so

$$
\chi(z) \leq \frac{C_{3, r_{1}} C_{1, r} C_{2}^{n+1}}{c_{1} r_{1}^{2 n}(1-r)^{n+1}} \frac{1}{\nu\left(B_{D}\left(z_{0}, r\right)\right)} \int_{B_{D}\left(z_{0}, R\right)} \chi d \nu
$$

\section{Carleson measures}

Let $D \subset \subset \mathbb{C}^{n}$ be a strongly pseudoconvex bounded domain in $\mathbb{C}^{n}$. Given $0<p<+\infty$ the Bergman space $A^{p}(D)$ of $D$ is the Banach space of holomorphic $L^{p}$-functions on $D$, that is $A^{p}(D)=L^{p}(D) \cap \mathcal{O}(D)$, endowed with the $L^{p}$-norm

$$
\|f\|_{p}^{p}=\int_{D}|f(z)|^{p} d \nu
$$

where $\nu$ is the Lebesgue measure normalized so that $\nu\left(B^{n}\right)=1$.

A finite positive Borel measure $\mu$ on $D$ is said to be a Carleson measure of $A^{p}(D)$ if there exists $C_{p}>0$ such that

$$
\forall f \in A^{p}(D) \quad \int_{D}|f(z)|^{p} d \mu \leq C_{p}\|f\|_{p}^{p} .
$$

As explained in the introduction, our aim is to give a characterization of Carleson measures involving the Bergman kernel of $D$.

Let $K: D \times D \rightarrow \mathbb{C}$ be the Bergman kernel of $D$ (see, e.g., [Kr, Section 1.4]); it has the reproducing property

$$
\forall f \in A^{2}(D) \forall z \in D \quad f(z)=\int_{D} K(z, \zeta) f(\zeta) d \nu .
$$

Since $K(\cdot, \zeta)=\overline{K(\zeta, \cdot)} \in A^{2}(D)$, in particular we have

$$
K(z, z)=\int_{D}|K(z, \zeta)|^{2} d \nu(\zeta)=\|K(z, \cdot)\|_{2}^{2} .
$$

For each $z_{0} \in D$ let $k_{z_{0}} \in A^{2}(D)$ be the normalized Bergman kernel given by

$$
k_{z_{0}}(z)=\frac{K\left(z, z_{0}\right)}{\left\|K\left(\cdot, z_{0}\right)\right\|_{2}}=\frac{K\left(z, z_{0}\right)}{\sqrt{K\left(z_{0}, z_{0}\right)}} ;
$$

clearly, $\left\|k_{z_{0}}\right\|_{2}=1$. The Berezin transform $B \mu$ of a finite measure $\mu$ on $D$ is the function given by

$$
B \mu(z)=\int_{D}\left|k_{z}(\zeta)\right|^{2} d \mu(\zeta)
$$

for all $z \in D$.

We recall the following estimate from above on the Bergman kernel:

Lemma 2.1: Let $D \subset \subset \mathbb{C}^{n}$ be a strongly pseudoconvex bounded domain. Then there exists $C_{4}>0$ such that

$$
\forall z_{0} \in D \quad\left|K\left(z_{0}, z_{0}\right)\right| \leq \frac{C_{4}}{d\left(z_{0}, \partial D\right)^{n+1}} .
$$

Proof: It follows immediately from [Hö, Theorem 3.5.1] or from [R, p. 186]. 
Our next result is an estimate from below on the Bergman kernel, valid close enough to the boundary.

Lemma 2.2: Let $D \subset \subset \mathbb{C}^{n}$ be a strongly pseudoconvex bounded domain. Then for every $r \in(0,1)$ there exist $c_{5, r}>0$ and $\delta_{r}>0$ such that if $z_{0} \in D$ satisfies $d\left(z_{0}, \partial D\right)<\delta_{r}$ then

$$
\forall z \in B_{D}\left(z_{0}, r\right) \quad\left|K\left(z, z_{0}\right)\right| \geq \frac{c_{5, r}}{d\left(z_{0}, \partial D\right)^{n+1}}
$$

Proof: Let $\psi$ be a defining function for $D$; in particular, there are $C_{6}, c_{6}>0$ such that

$$
c_{6} d(z, \partial D) \leq|\psi(z)| \leq C_{6} d(z, \partial D)
$$

in a neighbourhood of $\bar{D}$.

The main Theorem 2 in $[\mathrm{F}]$ implies that there is $\eta>0$ so that

$$
\left|K\left(z, z_{0}\right)\right| \geq \frac{c}{\left(\psi(z)+\psi\left(z_{0}\right)+\rho\left(z, z_{0}\right)\right)^{n+1}}
$$

for a suitable constant $c>0$ as soon as $d\left(z_{0}, \partial D\right)+d(z, \partial D)+\left\|z-z_{0}\right\|<\eta$, where

$$
\rho\left(z, z_{0}\right)=\left\|z-z_{0}\right\|^{2}+\left|\partial \psi_{z_{0}}\left(z-z_{0}\right)\right|
$$

Put

$$
\delta_{r}=\frac{\eta}{3} \min \left\{1, \frac{1-r}{C_{2}}, \frac{c_{2, r} \eta}{3}\right\} ;
$$

then Lemmas 1.2 and 1.4 imply that $d\left(z_{0}, \partial D\right)<\delta_{r}$ yields $d\left(z_{0}, \partial D\right)+d(z, \partial D)+\left\|z-z_{0}\right\|<\eta$ for all $z \in B_{D}\left(z_{0}, r\right)$. Using Lemmas 1.2 and 1.4 and (2.1) we then get that if $d\left(z_{0}, \partial D\right)<\delta_{r}$ then

$$
\begin{aligned}
\left|K\left(z, z_{0}\right)\right| & \geq \frac{c}{\psi\left(z_{0}\right)^{n+1}}\left(\frac{1}{1+\frac{\psi(z)}{\psi\left(z_{0}\right)}+\frac{\rho\left(z, z_{0}\right)}{\psi\left(z_{0}\right)}}\right)^{n+1} \\
& \geq \frac{c(1-r)^{n+1}}{C_{6}^{n+1} d\left(z_{0}, \partial D\right)^{n+1}}\left(\frac{c_{6}}{c_{6}+C_{2} C_{6}+1 / c_{2, r}}\right)^{n+1}
\end{aligned}
$$

for all $z \in B_{D}\left(z_{0}, r\right)$, and we are done.

Remark 2.1: When $D$ is strongly convex we can take $c_{5, r}=c_{5}\left(1-r^{2}\right)^{n+1}$ with $c_{5}>0$ independent of $r$.

As a corollary we get a crucial estimate from below for the normalized Bergman kernel:

Corollary 2.3: Let $D \subset \subset \mathbb{C}^{n}$ be a strongly pseudoconvex bounded domain. Then for every $r \in(0,1)$ there exist $c_{7, r}>0$ and $\delta_{r}>0$ such that if $z_{0} \in D$ satisfies $d\left(z_{0}, \partial D\right)<\delta_{r}$ then

$$
\forall z \in B_{D}\left(z_{0}, r\right) \quad\left|k_{z_{0}}(z)\right|^{2} \geq \frac{c_{7, r}}{d\left(z_{0}, \partial D\right)^{n+1}}
$$

Proof: It follows from Lemmas 2.1 and 2.2, with $c_{7, r}=c_{5, r}^{2} / C_{4}$.

Remark 2.2: When $D$ is strongly convex we can take $c_{7, r}=c_{7}\left(1-r^{2}\right)^{2(n+1)}$ with $c_{7}>0$ independent of $r$.

Now we can finally prove the promised characterization for the Carleson measures of $A^{p}(D)$ : 
Theorem 2.4: Let $\mu$ be a finite positive Borel measure on a strongly pseudoconvex bounded domain $D \subset \subset \mathbb{C}^{n}$. Then the following statements are equivalent:

(i) $\mu$ is a Carleson measure of $A^{p}(D)$ for some (and hence all) $p \in(0,+\infty)$;

(ii) the Berezin transform of $\mu$ is a bounded function;

(iii) for every $r \in(0,1)$ there exists $C_{r}>0$ such that $\mu\left(B_{D}\left(z_{0}, r\right)\right) \leq C_{r} \nu\left(B_{D}\left(z_{0}, r\right)\right)$ for all $z_{0} \in D$;

(iv) for some $r \in(0,1)$ there exists $C_{r}>0$ such that $\mu\left(B_{D}\left(z_{0}, r\right)\right) \leq C_{r} \nu\left(B_{D}\left(z_{0}, r\right)\right)$ for all $z_{0} \in D$.

Proof: (i) $\Longrightarrow$ (ii): By [CM] we can assume that $\mu$ is a Carleson measure of $A^{2}(D)$. Then

$$
B \mu\left(z_{0}\right)=\int_{D}\left|k_{z_{0}}(z)\right|^{2} d \mu(z) \leq C\left\|k_{z_{0}}\right\|_{2}^{2}=C
$$

for a suitable $C>0$, and $B \mu$ is bounded. we get

(ii) $\Longrightarrow\left(\right.$ iii): Fix $r \in(0,1)$. If $d\left(z_{0}, \partial D\right) \geq \delta_{r}$, where $\delta_{r}>0$ is given by Lemma 2.2, then using Lemma 1.1

$$
\mu\left(B_{D}\left(z_{0}, r\right)\right) \leq \mu(D) \leq \frac{\mu(D)}{c_{1} r^{2 n} \delta_{r}^{n+1}} \nu\left(B_{D}\left(z_{0}, r\right)\right)
$$

as desired.

Assume now $d\left(z_{0}, \partial D\right)<\delta_{r}$. Since the Berezin transform is bounded, there exists $C_{8}>0$ independent of $z_{0}$ and $r$ such that

$$
\int_{B_{D}\left(z_{0}, r\right)}\left|k_{z_{0}}(z)\right|^{2} d \mu(z) \leq B \mu\left(z_{0}\right) \leq C_{8} .
$$

Hence Corollary 2.3 yields

$$
\frac{c_{7, r}}{d\left(z_{0}, \partial D\right)^{n+1}} \mu\left(B_{D}\left(z_{0}, r\right)\right) \leq C_{8}
$$

Recalling Lemma 1.1 we get

$$
\mu\left(B_{D}\left(z_{0}, r\right)\right) \leq \frac{C_{8}}{c_{7, r}} d\left(z_{0}, \partial D\right)^{n+1} \leq \frac{C_{8}}{c_{1} c_{7, r} r^{2 n}} \nu\left(B_{D}\left(z_{0}, r\right)\right)
$$

and we are done in this case too.

(iii) $\Longrightarrow$ (iv): obvious.

(iv) $\Longrightarrow$ (i): It follows from Lemmas 1.1, 1.2, Corollary 1.7 and [Lu]; for the sake of completeness we give here a slightly different proof. Let $\left\{z_{k}\right\}$ be the sequence given by Lemma 1.5. Clearly

$$
\int_{D}|f(z)|^{p} d \mu(z) \leq \sum_{k=1}^{\infty} \int_{B_{D}\left(z_{k}, r\right)}|f(z)|^{p} d \mu(z)
$$

for all $f \in A^{p}(D)$. Since $|f|^{p}$ is plurisubharmonic and nonnegative, Corollary 1.8 and (v) yields

$$
\begin{aligned}
\int_{B_{D}\left(z_{k}, r\right)}|f(z)|^{p} d \mu(z) & \leq \frac{K_{r}}{\nu\left(B_{D}\left(z_{k}, r\right)\right)} \int_{B_{D}\left(z_{k}, r\right)} d \mu(z) \int_{B_{D}\left(z_{k}, R\right)}|f(\zeta)|^{p} d \nu(\zeta) \\
& =K_{r} \frac{\mu\left(B_{D}\left(z_{k}, r\right)\right)}{\nu\left(B_{D}\left(z_{k}, r\right)\right)} \int_{B_{D}\left(z_{k}, R\right)}|f(\zeta)|^{p} d \nu(\zeta) \\
& \leq K_{r} C_{r} \int_{B_{D}\left(z_{k}, R\right)}|f(\zeta)|^{p} d \nu(\zeta)
\end{aligned}
$$

where $R=\frac{1}{2}(1+r)$. Hence

$$
\int_{D}|f(z)|^{p} d \mu(z) \leq K_{r} C_{r} \sum_{k=1}^{\infty} \int_{B_{D}\left(z_{k}, R\right)}|f(\zeta)|^{p} d \nu(\zeta) \leq K_{r} C_{r} m\|f\|_{p}^{p}
$$

where $m$ is given by Lemma 1.5 , and so $\mu$ is a Carleson measure of $A^{p}(D)$.

Remark 2.3: Notice that (2.2) says that if $\mu$ is a Carleson measure then we can find $C>0$ so that

$$
\int_{D}|f(z)|^{p} d \mu(z) \leq C\|f\|_{p}^{p}
$$

for all $f \in A^{p}(D)$ and all $p \in(0,+\infty)$; in other words, the constant $C$ is independent of $p$. 


\section{Uniformly discrete sequences}

Let $(X, d)$ be a metric space. A sequence $\Gamma=\left\{x_{j}\right\} \subset X$ of points in $X$ is uniformly discrete if there exists $\delta>0$ such that $d\left(x_{j}, x_{k}\right) \geq \delta$ for all $j \neq k$. In this case $\inf _{j \neq k} d\left(x_{j}, x_{k}\right)$ is the separation constant of $\Gamma$.

Furthermore, given $x_{0} \in X, r>0$ and a subset $\Gamma \subset X$, we shall denote by $N\left(x_{0}, r, \Gamma\right)$ the number of points of $\Gamma$ contained in the ball of center $x_{0}$ and radius $r$.

We can use $N\left(x_{0}, r, \Gamma\right)$ to detect finite unions of uniformly discrete sequences:

Lemma 3.1: Let $X$ be a metric space, and $\Gamma=\left\{x_{n}\right\}_{n \in \mathbb{N}} \subset X$ a sequence in $X$. If there are $N \geq 1$ and $r>0$ such that $N(x, r, \Gamma) \leq N$ for all $x \in X$, then $\Gamma$ is the union of at most $N$ uniformly discrete sequences.

Proof: We shall define $N$ disjoint uniformly discrete sequences $\Gamma_{0}, \ldots, \Gamma_{N-1} \subset \Gamma$ so that $\Gamma=\Gamma_{0} \cup \cdots \cup \Gamma_{N-1}$. To do so, we start with $\Gamma_{0}=\cdots=\Gamma_{N-1}=\varnothing$ and, arguing by induction on $n$, we shall put each $x_{n}$ in a specified $\Gamma_{j}$. As a matter of notation, if $x_{i} \in \Gamma_{j}$ we shall write $m\left(x_{i}\right)=j$, and we shall denote by $B(x, r)$ the metric ball of center $x$ and radius $r$.

Put $x_{0} \in \Gamma_{0}$. Assume we have already defined $m\left(x_{i}\right)$ for $i \leq n$, and consider $x_{n+1}$. By assumption, $\Gamma \cap B\left(x_{n+1}, r\right)$ contains at most $N$ points, one of which is $x_{n+1}$. Hence $\left\{x_{0}, \ldots, x_{n}\right\} \cap B\left(x_{n+1}, r\right)$ contains at most $N-1$ points, and we can define

$$
m\left(x_{n+1}\right)=\min \left\{i \in\{0, \ldots, N-1\} \mid i \neq m\left(x_{j}\right) \text { for all } 0 \leq j \leq n \text { such that } x_{j} \in B\left(x_{n+1}, r\right)\right\} .
$$

In this way $d\left(x_{n+1}, x_{j}\right) \geq r$ for all $x_{j} \in \Gamma_{m\left(x_{n+1}\right)}$ with $j<n+1$.

It now follows easily that $\Gamma_{0}, \ldots, \Gamma_{N-1}$ are uniformly discrete sequences with separation constant at least $\delta=r$, because by construction if $x_{h}, x_{k} \in \Gamma_{j}$ with $h>k$ we have $d\left(x_{h}, x_{k}\right) \geq r$.

We are now able to prove that uniformly discrete sequences give examples of Carleson measures:

Theorem 3.2: Let $D \subset \subset \mathbb{C}^{n}$ be a strongly pseudoconvex bounded domain, considered as a metric space with the distance $\rho_{D}=\tanh k_{D}$. Let $\Gamma=\left\{z_{j}\right\}_{j \in \mathbb{N}}$ be a sequence in $D$. Then the following statements are equivalent:

(i) $\Gamma$ is a finite union of uniformly discrete sequences;

(ii) $\sup N\left(z_{0}, r, \Gamma\right)<+\infty$ for some $r \in(0,1)$;

$z_{0} \in D$

(iii) $\sup N\left(z_{0}, r, \Gamma\right)<+\infty$ for all $r \in(0,1)$;

$z_{0} \in D$

(iv) there exists $p \in(0,+\infty)$ such that $\sum_{z_{j} \in \Gamma} d\left(z_{j}, \partial D\right)^{n+1} \delta_{z_{j}}$ is a Carleson measure of $A^{p}(D)$, where $\delta_{z_{j}}$ is the Dirac measure in $z_{j}$;

(v) there exists $C_{9}>0$ such that for all $p \in(0,+\infty)$ we have

$$
\forall f \in A^{p}(D) \quad \sum_{z_{j} \in \Gamma} d\left(z_{j}, \partial D\right)^{n+1}\left|f\left(z_{j}\right)\right|^{p} \leq C_{9}\|f\|_{p}^{p}
$$

in particular, $\sum_{z_{j} \in \Gamma} d\left(z_{j}, \partial D\right)^{n+1} \delta_{z_{j}}$ is a Carleson measure of $A^{p}(D)$ for all $p \in(0,+\infty)$.

Proof: $(\mathrm{v}) \Longrightarrow(\mathrm{iv})$ : Obvious.

(iv) $\Longrightarrow$ (iii): Fix $r \in(0,1)$, and let $\delta_{r}>0$ be given by Lemma 2.2. By Lemma 1.2, if $d\left(z_{0}, \partial D\right) \geq \delta_{r}$ then $z \in B_{D}\left(z_{0}, r\right)$ implies $d(z, \partial D) \geq \frac{1}{C_{2}}(1-r) \delta_{r}$. Using (iv) it is easy to see that only a finite number of $z_{j} \in \Gamma$ can have $d\left(z_{j}, \partial D\right) \geq \frac{1}{C_{2}}(1-r) \delta_{r}$; therefore to get (iii) it suffice to prove that the supremum is finite when $d\left(z_{0}, \partial D\right)<\delta_{r}$.

Given $z_{0} \in D$ with $d\left(z_{0}, \partial D\right)<\delta_{r}$, Corollary 2.3 and Lemma 1.2 yield

$$
\forall z \in B_{D}\left(z_{0}, r\right) \quad d(z, \partial D)^{n+1}\left|k_{z_{0}}(z)\right|^{2} \geq \frac{c_{7, r}}{C_{2}^{n+1}}(1-r)^{n+1} .
$$

By $[\mathrm{CM}]$ we can assume $p=2$; hence

$$
N\left(z_{0}, r, \Gamma\right) \leq \frac{C_{2}^{n+1}}{c_{7, r}(1-r)^{n+1}} \sum_{z \in B_{D}\left(z_{0}, r\right) \cap \Gamma} d(z, \partial D)^{n+1}\left|k_{z_{0}}(z)\right|^{2} \leq \frac{C_{2}^{n+1} C}{c_{7, r}(1-r)^{n+1}}\left\|k_{z_{0}}\right\|_{2}^{2}=\frac{C_{2}^{n+1} C}{c_{7, r}(1-r)^{n+1}}
$$


for a suitable $C>0$, as desired.

(iii) $\Longrightarrow$ (ii): Obvious.

(ii) $\Longrightarrow$ (i): Lemma 3.1 .

(i) $\Longrightarrow(v)$ : Clearly it suffices to prove the assertion when $\Gamma$ is a single uniformly discrete sequence. Let $\delta>0$ be the separation constant of $\Gamma$, and put $r=\delta / 2$. By the triangle inequality, the Kobayashi balls $B_{D}\left(z_{j}, r\right)$ are pairwise disjoint. Hence

$$
\int_{D}|f(z)|^{p} d \nu \geq \sum_{z_{j} \in \Gamma} \int_{B_{D}\left(z_{j}, r\right)}|f(z)|^{p} d \nu
$$

Now, $|f|^{p}$ is plurisubharmonic and nonnegative; hence Corollary 1.7 and Lemma 1.1 yield

$$
\int_{B_{D}\left(z_{j}, r\right)}|f(z)|^{p} d \nu \geq \frac{1}{C_{3, r}} \nu\left(B_{D}\left(z_{j}, r\right)\right)\left|f\left(z_{j}\right)\right|^{p} \geq \frac{c_{1}}{C_{3, r}} r^{2 n} d\left(z_{j}, \partial D\right)^{n+1}\left|f\left(z_{j}\right)\right|^{p}
$$

for all $z_{j} \in \Gamma$. Setting $C_{9}=\frac{C_{3, r}}{c_{1} r^{2 n}}$, the assertion follows.

Now we would like to study how fast a uniformly discrete sequence can escape to the boundary. A first result in this vein is an immediate corollary of the previous theorem:

Corollary 3.3: Let $\Gamma=\left\{z_{j}\right\} \subset D$ be a uniformly discrete sequence in a strongly pseudoconvex bounded domain $D \subset \subset \mathbb{C}^{n}$. Then

$$
\sum_{z_{j} \in \Gamma} d\left(z_{j}, \partial D\right)^{n+1}<+\infty
$$

Proof: It suffices to take $f \equiv 1$ in Theorem 3.2.(v).

The next result gives a worse estimate, but valid in any hyperbolic domain with finite Euclidean volume.

Proposition 3.4: Let $D \subset \mathbb{C}^{n}$ be a hyperbolic domain with finite Euclidean volume, endowed with the distance $\rho_{D}=\tanh k_{D}$. Let $\Gamma=\left\{z_{j}\right\} \subset D$ be an uniformly discrete sequence with $d\left(z_{j}, \partial D\right)<1$ for all $z_{j} \in \Gamma$. Then

$$
\sum_{z_{j} \in \Gamma} d\left(z_{j}, \partial D\right)^{2 n} h\left(-\frac{1}{\log d\left(z_{j}, \partial D\right)}\right)<+\infty
$$

for any increasing function $h: \mathbb{R}^{+} \rightarrow \mathbb{R}^{+}$such that

$$
\sum_{m=1}^{+\infty} h\left(\frac{1}{m}\right)<+\infty
$$

Proof: For $m \in \mathbb{N}$ put

$$
\Omega_{m}=\left\{z \in D \mid m<\log \frac{1}{d(z, \partial D)} \leq m+1\right\}=\left\{z \in D \mid e^{-(m+1)} \leq d(z, \partial D)<e^{-m}\right\} .
$$

Set $\Gamma_{m}=\Gamma \cap \Omega_{m}$; by assumption, $\Gamma=\bigcup_{m=0}^{+\infty} \Gamma_{m}$. Let $\delta>0$ be the separation constant of $\Gamma$, and put $r=\delta / 2$. The Kobayashi balls $B_{D}\left(z_{j}, r\right)$ centered in points of $\Gamma$ are pairwise disjoint; in particular,

$$
\sum_{z_{j} \in \Gamma_{m}} \nu\left(B_{D}\left(z_{j}, r\right)\right) \leq \nu(D)
$$

If $z \in D$ then the euclidean ball $B$ of center $z$ and radius $d(z, \partial D)$ is contained in $D$. But then $B_{D}(z, r)$ contains the Kobayashi ball $B_{B}(z, r)$ of $B$ centered in $z$ and of radius $r$, which is an euclidean ball of radius $r d(z, \partial D)$. Thus

$$
\nu\left(B_{D}\left(z_{j}, r\right)\right) \geq r^{2 n} d\left(z_{j}, \partial D\right)^{2 n} .
$$


Let $N_{m}$ be the cardinality of $\Gamma_{m}$. Then (3.1) yields

$$
N_{m} \leq \nu(D) r^{-2 n} e^{2 n(m+1)}=C_{9} e^{2 n m},
$$

for a suitable constant $C_{9}>0$ independent of $m$. Therefore

$$
\begin{aligned}
\sum_{z_{j} \in \Gamma} d\left(z_{j}, \partial D\right)^{2 n} h\left(-\frac{1}{\log d\left(z_{j}, \partial D\right)}\right) & =\sum_{m=0}^{+\infty} \sum_{z_{j} \in \Gamma_{m}} d\left(z_{j}, \partial D\right)^{2 n} h\left(-\frac{1}{\log d\left(z_{j}, \partial D\right)}\right) \\
& \leq \sum_{z_{j} \in \Gamma_{0}} d\left(z_{j}, \partial D\right)^{2 n} h\left(-\frac{1}{\log d\left(z_{j}, \partial D\right)}\right)+\sum_{m=1}^{+\infty} N_{m} e^{-2 m n} h\left(\frac{1}{m}\right) \\
& \leq C_{10}+C_{9} \sum_{m=1}^{+\infty} h\left(\frac{1}{m}\right)<+\infty .
\end{aligned}
$$

Remark 3.1: If $D \subset \subset \mathbb{C}^{n}$ is bounded and $\Gamma=\left\{z_{j}\right\} \subset D$ is an uniformly discrete (with respect to any distance inducing the natural topology of $D$ ) sequence, then $d\left(z_{j}, \partial D\right)<1$ for all but a finite number of elements of $\Gamma$.

To get the sharp estimate valid in strongly pseudoconvex bounded domains, we replace the Euclidean measure by the Eisenman-Kobayashi invariant measure (but see Remark 3.2 below).

For simplicity, let us recall the definition of the Eisenman-Kobayashi measure in domains of $\mathbb{C}^{n}$ only. Let $D \subset \mathbb{C}^{n}$ be a domain; the Eisenman-Kobayashi density $K_{D}: D \rightarrow \mathbb{R}^{+}$is given by

$$
K_{D}\left(z_{0}\right)=\inf \left\{\left|\operatorname{Jac}_{\mathbb{R}} f(O)\right|^{-1} \mid f: B^{n} \rightarrow D \text { holomorphic, } f(O)=z_{0}, d f_{O} \text { invertible }\right\} .
$$

It is not difficult to prove that $K_{D}$ is upper semicontinuous (see, e.g., [A, Proposition 2.3.37]); then the Eisenman-Kobayashi measure of $D$ is just $\tilde{\kappa}_{D}=K_{D} \nu$. When $D=B^{n}$, the Eisenman-Kobayashi measure coincides with the Bergman (or hyperbolic) volume (see, e.g., [A, Proposition 2.3.36]).

The next lemma contains an estimate on the Eisenman-Kobayashi measure of Kobayashi balls.

Lemma 3.5: Let $D \subset \subset \mathbb{C}^{n}$ be a strongly pseudoconvex bounded domain,. Then there exist $0<c_{11}, C_{11}$ such that

$$
\forall z_{0} \in D \forall r \in(0,1) \quad c_{11} r^{2 n}(1-r)^{n+1} \leq \tilde{\kappa}_{D}\left(B_{D}\left(z_{0}, r\right)\right) \leq \frac{C_{11}}{d\left(z_{0}, \partial D\right)^{n}(1-r)^{n}} .
$$

Proof: The estimates in $[\mathrm{M}]$ show that there are two constants $c_{12}, C_{12}>0$ such that

$$
\forall z \in D \quad \frac{c_{12}}{d(z, \partial D)^{n+1}} \leq K_{D}(z) \leq \frac{C_{12}}{d(z, \partial D)^{n+1}} .
$$

In particular, Lemmas 1.2 and 1.1 yield

$$
\begin{aligned}
\tilde{\kappa}_{D}\left(B_{D}\left(z_{0}, r\right)\right) & =\int_{B_{D}\left(z_{0}, r\right)} K_{D}(z) d \nu \geq c_{12} \int_{B_{D}\left(z_{0}, r\right)} \frac{1}{d(z, \partial D)^{n+1}} d \nu \geq \frac{c_{12}(1-r)^{n+1}}{C_{2}^{n+1} d\left(z_{0}, \partial D\right)^{n+1}} \nu\left(B_{D}\left(z_{0}, r\right)\right) \\
& \geq \frac{c_{12} c_{1}}{C_{2}^{n+1}} r^{2 n}(1-r)^{n+1} .
\end{aligned}
$$

For the upper estimate, notice that since $\partial D$ is and bounded there is an $\varepsilon>0$ such that, setting

$$
U_{\varepsilon}=D \backslash K_{\varepsilon}=\{z \in D \mid d(z, \partial D)<\varepsilon\},
$$

then there exists a smooth diffeomorphism $\Psi: \bar{U}_{\varepsilon} \rightarrow[0, \varepsilon] \times \partial D$ with $\Psi^{-1}(\{t\} \times \partial D)=\{z \in D \mid d(z, \partial D)=t\}$ for all $t \in(0, \varepsilon]$. 
Now Lemma 1.2 implies

$$
B_{D}\left(z_{0}, r\right) \subset\{z \in D \mid d(z, \partial D) \geq l(r)\}
$$

where $l(r)=\frac{1}{C_{2}}(1-r) d\left(z_{0}, \partial D\right)$. Thus, denoting by $\sigma$ the usual $(2 n-1)$-measure on $\partial D$, and by $\lambda$ the product measure on $[0, \varepsilon] \times \partial D$, and using the compactness of $\bar{U}_{\varepsilon}$, we have

$$
\begin{aligned}
\tilde{\kappa}_{D}\left(B_{D}\left(z_{0}, r\right)\right) & =\int_{B_{D}\left(z_{0}, r\right)} K_{D}(z) d \nu \leq C_{12} \int_{B_{D}\left(z_{0}, r\right)} \frac{1}{d(z, \partial D)^{n+1}} d \nu \\
& \leq C_{12} \int_{K_{\epsilon}} \frac{1}{d(z, \partial D)^{n+1}} d \nu+C_{12} \int_{B_{D}\left(z_{0}, r\right) \cap U_{\epsilon}} \frac{1}{d(z, \partial D)^{n+1}} d \nu \\
& \leq C_{13}+C_{14} \int_{\Psi\left(B_{D}\left(z_{0}, r\right) \cap U_{\epsilon}\right)} \frac{1}{t^{n+1}} d \lambda \leq C_{13}+C_{14} \int_{[l(r), \varepsilon] \times \partial D} \frac{1}{t^{n+1}} d \lambda \\
& \leq C_{13}+C_{14} \sigma(\partial D)\left(\frac{1}{l(r)^{n}}-\frac{1}{\epsilon^{n}}\right) \leq \frac{C_{15}}{l(r)^{n}}=\frac{C_{2}^{n} C_{15}}{d\left(z_{0}, \partial D\right)^{n}(1-r)^{n}}
\end{aligned}
$$

for suitable constants $C_{13}, C_{14}, C_{15}>0$ independent of $z_{0}$ and $r$.

Remark 3.2: We stated this lemma in terms of the Eisenman-Kobayashi measure just to keep with the invariant approach of this paper; but for the purpose of the next theorem any measure providing the same estimates would work. For instance, we might use the measure $\mu=d(\cdot, \partial D)^{-(n+1)} \nu$.

We are now able to prove the promised sharp estimate for strongly pseudoconvex domains:

Theorem 3.6: Let $D \subset \subset \mathbb{C}^{n}$ be a strongly pseudoconvex bounded domain, endowed with the distance $\rho_{D}=\tanh k_{D}$. Let $\Gamma=\left\{z_{j}\right\} \subset D$ be an uniformly discrete sequence with $d\left(z_{j}, \partial D\right)<1$ for all $z_{j} \in \Gamma$. Then

$$
\sum_{z_{j} \in \Gamma} d\left(z_{j}, \partial D\right)^{n} h\left(-\frac{1}{\log d\left(z_{j}, \partial D\right)}\right)<+\infty
$$

for any increasing function $h: \mathbb{R}^{+} \rightarrow \mathbb{R}^{+}$such that

$$
\sum_{m=1}^{+\infty} h\left(\frac{1}{m}\right)<+\infty
$$

Proof: Fix $z_{0} \in D$, and for $m \in \mathbb{N}$ set

$$
D_{m}=\left\{z \in D \mid \frac{m}{2} \leq k_{D}\left(z_{0}, z\right)<\frac{m+1}{2}\right\},
$$

and let $\Gamma_{m}=D_{m} \cap \Gamma$. Let $\delta>0$ be the separation constant of $\Gamma$ (with respect to $k_{D}$ ), and let $r=\tanh (\delta / 2)$. Clearly, if $z_{j} \in \Gamma_{m}$ and $z \in B_{D}\left(z_{j}, r\right)$, then $k_{D}\left(z_{0}, z\right)<\frac{1}{2}(m+1+\delta)$. Since, as usual, the Kobayashi balls $B_{D}\left(z_{j}, r\right)$ are pairwise disjoint, using Lemma 3.5 we get

$$
c_{11} r^{2 n}(1-r)^{n+1} N_{m} \leq \sum_{z_{j} \in \Gamma_{m}} \tilde{\kappa}_{D}\left(B_{D}\left(z_{j}, r\right)\right) \leq \tilde{\kappa}_{D}\left(B_{D}\left(z_{0}, R_{m}\right)\right) \leq \frac{C_{11}}{d\left(z_{0}, \partial D\right)^{n}\left(1-R_{m}\right)^{n}},
$$

where $R_{m}=\tanh \frac{m+1+\delta}{2}$; hence there is $C_{16}>0$ (depending on $z_{0}$ and $\delta$ but not on $m$ ) such that

$$
N_{m} \leq C_{16} e^{m n}
$$

Now, the estimates (1.1) on the boundary behavior of the Kobayashi distance yield $C_{17}, C_{18}>0$ such that

$$
d(z, \partial D) \leq C_{17} \exp \left(-2 k_{D}\left(z_{0}, z\right)\right)
$$


and

$$
-\frac{1}{\log d(z, \partial D)} \leq \frac{1}{2 k_{D}\left(z_{0}, z\right)-C_{18}}
$$

as soon as $2 k_{D}\left(z_{0}, z\right)>C_{18}$. In particular, if $z_{j} \in \Gamma_{m}$ we have

$$
d\left(z_{j}, \partial D\right) \leq C_{17} e^{-m} \quad \text { and } \quad-\frac{1}{\log d(z, \partial D)} \leq \frac{1}{m-C_{18}} .
$$

as soon as $m>C_{18}$. Then

$$
\begin{aligned}
& \sum_{z_{j} \in \Gamma} d\left(z_{j}, \partial D\right)^{n} h\left(-\frac{1}{\log d\left(z_{j}, \partial D\right)}\right)=\sum_{m=0}^{+\infty} \sum_{z_{j} \in \Gamma_{m}} d\left(z_{j}, \partial D\right)^{n} h\left(-\frac{1}{\log d\left(z_{j}, \partial D\right)}\right) \\
& \leq C_{19}+C_{17}^{n} \sum_{m=m_{0}}^{+\infty} N_{m} e^{-n m} h\left(\frac{1}{m-C_{18}}\right) \leq C_{19}+C_{17}^{n} C_{16} \sum_{m=0}^{+\infty} h\left(\frac{1}{m}\right)<+\infty
\end{aligned}
$$

where $m_{0}$ is the smallest integer greater than $C_{18}$.

In particular, taking $h(x)=e^{-1 / x}$ we recover Corollary 3.3 .

Remark 3.3: The statement of Theorem 3.6 is sharp. In fact, MacCluer $[\mathrm{Mc}]$ has constructed for any increasing function $h: \mathbb{R}^{+} \rightarrow \mathbb{R}^{+}$with $\lim _{t \rightarrow 0^{+}} h(t)=0$ and $\sum_{m=1}^{\infty} h(1 / m)=+\infty$ an uniformly discrete sequence $\Gamma=\left\{z_{j}\right\}$ in $B^{n}$ such that

$$
\sum_{z_{j} \in \Gamma} d\left(z_{j}, \partial B^{n}\right)^{n} h\left(-\frac{1}{\log d\left(z_{j}, \partial B^{n}\right)}\right)=+\infty
$$

\section{References}

[A] M. Abate: Iteration theory of holomorphic maps on taut manifolds. Mediterranean Press, Cosenza, 1989; see http://www.dm.unipi.it/ abate/libri/libriric/libriric.html.

[C] L. Carleson: Interpolations by bounded analytic functions and the corona problem. Ann. of Math. 76 (1962), 547-559.

[CM] J.A. Cima, P.R. Mercer: Composition operators between Bergman spaces on convex domains in $\mathbb{C}^{n}$. J. Operator Theory 33 (1995), 363-369.

[CW] J.A. Cima, W.R. Wogen: A Carleson measure theorem for the Bergman space on the ball. J. Operator Theory 7 (1982), 157-165.

[D] P.L. Duren: Extension of a theorem of Carleson. Bull. Amer. Math. Soc. 75 (1969), 143-146.

[DS] P.L. Duren, A. Schuster: Bergman spaces. American Mathematical Society, Providence, RI, 2004.

[DSV] P.L. Duren, A. Schuster, D. Vukotić: On uniformly discrete sequences in the disk. In Quadrature domains and applications, P. Ebenfeld, B. Gustafsson, D. Khavinson and M. Putinar eds., Birkhäuser, Basel, 2005, pp. 131-150.

[DW] P.L. Duren, R. Weir: The pseudohyperbolic metric and Bergman spaces in the ball. Trans. Amer. Math. Soc. 359 (2007), 63-76.

[F] C. Fefferman: The Bergman kernel and biholomorphic mappings of pseudoconvex domains. Invent. Math. 26 (1974), 1-65.

$[\mathrm{H}] \quad$ W.W. Hastings: A Carleson measure theorem for Bergman spaces. Proc. Amer. Math. Soc. 52 (1975), 237-241.

[Hö] L. Hörmander: An introduction to complex analysis in several variables. North Holland, Amsterdam, 1973.

[JMT] M. Jevtić, X. Massaneda, P.J. Thomas: Interpolating sequences for weighted Bergman spaces of the ball. Michigan Math. J. 43 (1996), 495-517. 
[JP] M. Jarnicki, P. Pflug: Invariant distances and metrics in complex analysis. Walter de Gruyter \& co., Berlin, 1993.

[K] S. Kobayashi: Hyperbolic complex spaces. Springer-Verlag, Berlin, 1998.

[Kr] S. Krantz: Function theory of several complex variables. Wiley, New York, 1981.

[KM] S.G. Krantz, D. Ma: Bloch functions on strongly pseudoconvex domains. Indiana Univ. Math. J. 37 (1988), 145-163.

[Li] H. Li: BMO, VMO and Hankel operators on the Bergman space of strictly pseudoconvex domains. J. Funct. Anal. 106 (1992), 375-408.

[Lu] D. Luecking: A technique for characterizing Carleson measures on Bergman spaces. Proc. Amer. Math. Soc. 87 (1983), 656-660.

[M] D. Ma: Boundary behavior of invariant metrics and volume forms on strongly pseudoconvex domains. Duke Math. J. 63 (1991), 673-697.

[Mc] B.D. MacCluer: Uniformly discrete sequences in the ball. J. Math. Anal. Appl. 318 (2006), 37-42.

[Ma] X. Massaneda: $A^{-p}$ interpolation in the unit ball. J. London Math. Soc. 52 (1995), 391-401.

[O] V.L. Oleinik: Embeddings theorems for weighted classes of harmonic and analytic functions. J. Soviet Math. 9 (1978), 228-243.

[OP] V.L. Oleinik, B.S. Pavlov: Embedding theorems for weighted classes of harmonic and analytic functions. J. Soviet Math. 2 (1974), 135-142.

[R] R.M. Range: Holomorphic functions and integral representations in several complex variables. Springer-Verlag, Berlin, 1986.

[Ru] W. Rudin: Function theory in the unit ball of $\mathbb{C}^{n}$. Springer-Verlag, Berlin, 1980. 